\title{
Epidemiological Study of Brain Tumours Incidence in Sudan During 2014-2017
}

\author{
Mohammed Ahmed Ali Omer ${ }^{1,2, *}$, Ali Bashir Ali Elhaj ${ }^{2}$ \\ ${ }^{1}$ College of Applied Medical Science, Department of Radiologic Technology, Qassim University, Buraidah, KSA \\ ${ }^{2}$ Department of Radiotherapy \& Nuclear Medicine, College of Medical Radiologic Science, Sudan University of Science and Technology, \\ Khartoum, Sudan
}

Email address:

alkajam@gmail.com (M. A. A. Omer), tamboulali78@yahoo.com (A. B. A. Elhaj)

${ }^{*}$ Corresponding author

To cite this article:

Mohammed Ahmed Ali Ome, Ali Bashir Ali Elhaj. Epidemiological Study of Brain Tumours Incidence in Sudan during 2014-2017. Journal of Health and Environmental Research. Vol. 3, No. 4, 2017, pp. 57-61. doi: 10.11648/j.jher.20170304.11

Received: July 13, 2017; Accepted: August 3, 2017; Published: September 26, 2017

\begin{abstract}
The aim of this study was to highlight the epidemiology of brain tumors (BTs) in Sudan during 2014-2017 as retrospective and surveyed questionnaire collected data method. The data (annual incidence, frequencies of BTs based on gender and age, anatomical location, pathologies and geographical distribution) analyzed using EXCEL software which revealed that: BTs increase annually by a factor of $7.2 \%$ and predominant among male with $57.1 \%$. The involved anatomical brain sites were midbrain 35\%, left brain (30\%) and right brain (24\%). BTs observed among age groups of 1-10 years old for both gender with increasing similar trend incidence following aging which peaking at 55-65 years old. The common pathologies of brain were atrophy and infarction that represents $13.8 \%$ and $7.2 \%$ respectively and benign and malignant tumors represented (19.8\%) and (34.4\%) respectively. The most endemic sectors by BTs were central and Northern of Sudan that represented $45 \%$ and $35 \%$ respectively, then eastern and southern of Sudan that represented $12 \%$ and $8 \%$ respectively.
\end{abstract}

Keywords: Brain-Tumors, Epidemiology, Incidence, Radiation

\section{Introduction}

Brain tumors BTs (benign or malignant) are originate as intracranial masses with heterogeneous features and serious consequences with bad prognosis which representing about $2 \%$ of all adult tumors [1]. They could involve all ages, however their incidence increases at third decade and peaking by 65 onwards among both gender with slightly high incidence among male $[2,3]$. BTs have been as leading cause of mortality among children and as third causative death among young adults aged 15 to 34 years old [4]. Regarding BTs incidence, Stefan et al, [5] stated that: the general incidence of intracerebral tumors represent 8.4-11.8 for men and 5.8-9.3 for women with annual increment of $0.6 \%$ for men and $0.9 \%$ for women. However in Sudan the only study focus in BTs was carried by Hussein et al, [6] in which they stated that: there were 123 cases of BTs over ten years recorded during 1971-1981 at Shaab Teaching Hospital in Khartoum. However Intisar et al, [7] had focus in general cancer incidence in Khartoum-Sudan during 2009-2010; during which 6771 patients were registered as new cancer cases with $53.8 \%$ cases in women and $46.2 \%$ in men, and BTs have been noted among patients under 15 years old rated as (0.9, Rate: per 100000). Same trend of cancer survey in Sudan during 2009-2014 carried out by Mohamed et al, [8] stated that the most five common type among children were leukaemia, lymphoma, eye tumors, sarcoma and brain tumors in children, with a median involved age of 10-20 years old higher in men. And in relation with Sudan state; Northern Sudan, River Nile and Khartoum revealed up to 8-fold higher cancer incidence rates than Al Gedarif, Southern Dafur and Blue Nile with considerable high incidence of esophageal cancer in Kassala (Eastern Sudan).

The causative factors play a major role for epidemiological distribution in Sudan and many countries. However the blamed risk factors predominantly Sudanese population exposed to or practicing them are: the trend of civilize fast food and processed meat that could carries nitrates $\backslash$ nitrite compounds, depending on underground water which is 
polluted with sewage, shanty and randomly living where people dowelled near electric power transformer and towers, and antennas of wireless communication, rare hereditary syndromes, therapeutic radiation, and immune suppression giving rise to lymphomas [9] in addition to cosmic radiation exposure at high altitude, aging, gender, cultural, ethnic, or geographic differences in risk factors [10]. In this realm; Mackinny [11] summarized the risk factors of BTs and the evaluated risks were (Ionizing radiation, Mobile phone, Extremely low frequency electromagnetic fields, Specific infections, Allergies, and Occupations).

Epidemiological studies is a science that highlight the distribution and correlation of endemic diseases with risk factors to contribute in communities' protection and facilitate the interpretation of future trends of diseases [12] i.e. correlates between the origin of diseases and causes among the communities which depends on epidemiologists, laboratory scientists, statisticians, physicians, health care providers, and public health professionals to get to the root of health problems and outbreaks in a community. The aim of current study is to show the BTs distribution in Sudan with incidence $\%$ among gender, age groups, anatomical location and the common types of histopathology relative to handed risk factors observed in Sudan sectors.

\section{Methodology}

The following as Experimental \& retrospective study for 350 BT patients who referred for magnetic Resonance spectroscopy MRS at Radiology department at Royal Care hospital (RCH), Radiation and Isotopes Center of Khartoum (RICK) and Alshaab Hospitals in Sudan during 2014-2017.

MRS (single or multi-voxel technique) is noninvasive diagnostic procedure for brain metabolite that could register the pattern of tissue with chemical compounds (Choline compounds (Cho), Creatine and Phosphocreatine (Cr), $\mathrm{N}$ Acetyl-Aspartate (NAA), and Lactate (Lac)) and map out the spatial distribution of metabolites within the brain.

The collected variables from the total sample size were the incidence $\%$ based on gender, age, anatomical location involved by BTs\%, geographical distribution of BTs $\%$ and brain pathologies\% depending on diagnostic findings (High Choline indicate brain malignancy, weak NAA signal refers to replacement of healthy brain tissue by tumor cells, level of $\mathrm{Cr}$ refers to high energy metabolism and Lac refers to anaerobic metabolism). Also a survey questionnaire for 350 houses to reveal the utilization \% of underground water $(75 \%)$ and toilets or water closed with drilled pipe to underground water $(65 \%)$. The data have been analyzed using EXCELL software inform of bars and scattered plot.

\section{Result}

The following results highlighting the BTs in Sudan during the period of 2014-2017, incidence $\%$ based on gender, anatomical location, and variation incidence with aging, types of brain histopathology and the involved regions.

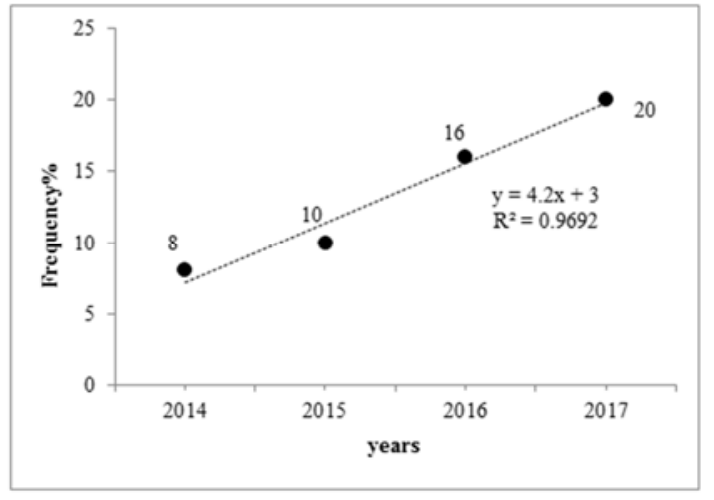

Figure 1. Shows the incidence\% of Brain tumors during the period 2014 2017.

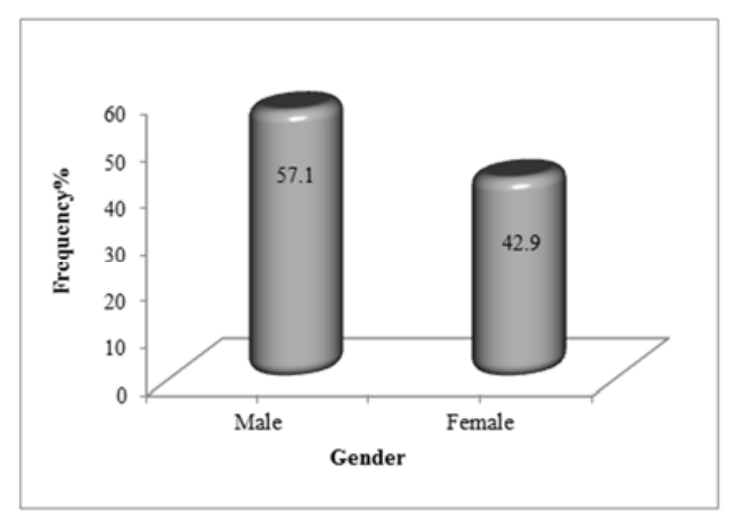

Figure 2. Shows the brain tumors incidence \% in Sudanese population during 2014-2017.

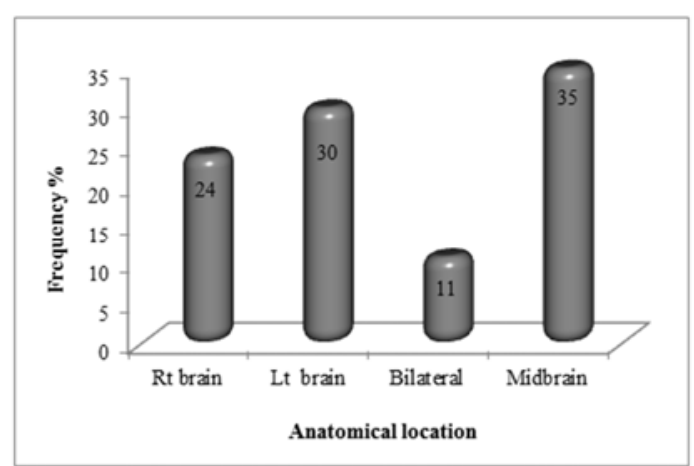

Figure 3. Shows the anatomical sites involved by brain tumors during 20142017 in Sudan.

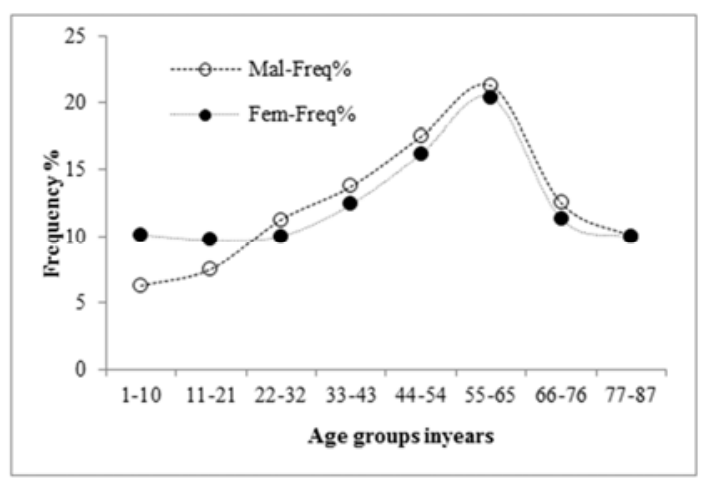

Figure 4. Shows the brain tumors distribution in Sudanese population based on age group during 2014-2017. 


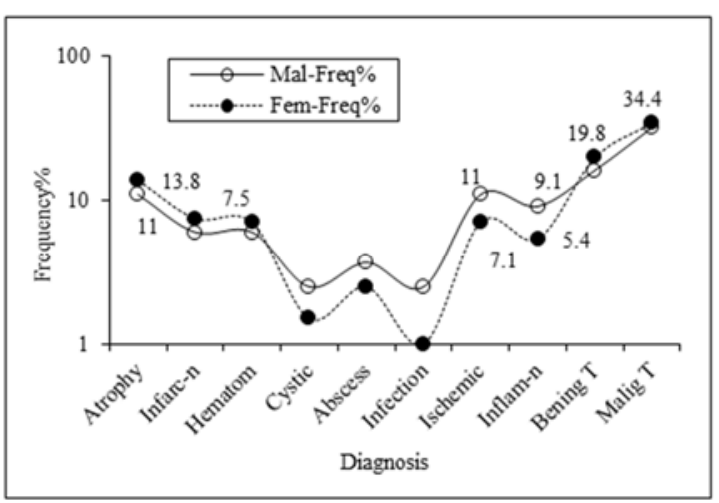

Figure 5. Shows the types of brain pathologies in Sudanese population during 2014-2017.

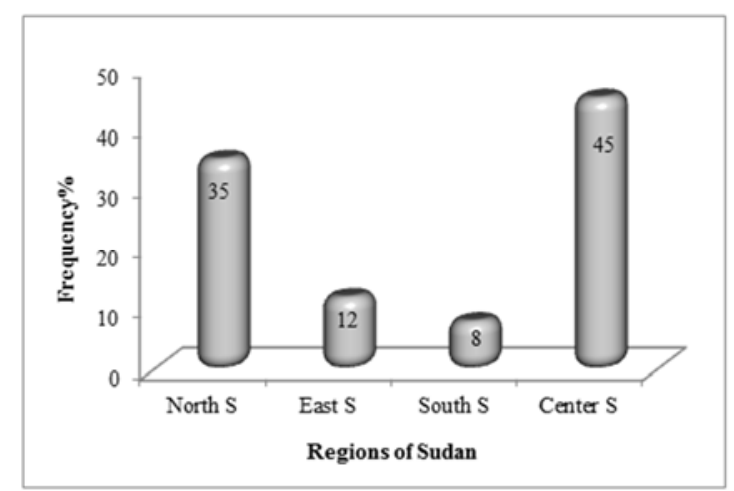

Figure 6. Shows the distribution of brain tumors in different geographical sectors of Sudan $(S)$.

\section{Discussion \& Analysis}

The incidence $\%$ of Brain tumors during the period 2014 2017, presented the total tumors (benign \& malignant) incidence $(54 \%)$ from the total sample pathologies among 350 patients (Figure 1). It reveals clearly there was an increasing incidence annually by a factor of 7.2. Such results is quiet agreed with Greig et al, [13] who reported that: the annual percentage increment of primary brain tumors among age groups $(75-79,80-84$, and 85$)$ were $+7.0 \%,+20.4 \%$, and $+23.4 \%$ respectively. And it has been as a matter of debate whether such high incidence of diagnosed BTs ascribed to actual increased BTs incidence among the universe population or due to rapid diagnostic technologies development, but in Sudan the risk and etiological factors are present and the populations have been involving deeply in such factors.

The survey of brain tumors incidence\% in Sudanese population during 2014-2017, revealed that: the male were the most gender involved by brain tumors as $57.1 \%$ relative to female (Figure 2). The high incidence of brain tumors among male ascribed to retinoblastoma protein (RB), which reducing cancer risk among male due to it is less activity and on the other hand the female are fewer incidences of brain tumors due to increase in active or less of retinoblastoma protein $[14,15]$. As general BTs incidence increment has been ascribed to genetic factors accumulation (inclusive family marriage) and inherited altered genes (EGFR amplification and mutation, amplification of CDK4 or $M D M 2$, and deletion or mutation of TP53, RB, or PTEN) which controlling cell cycle $[16,10]$. In comparison with previous study; CBTRUS, [17] also highlighted that: the brain tumors are so predominant among male with high incident in developed countries (males, 5.8 and females, 4.1 per 100,000) than in less developed countries (males 3.0 and females 2.1 per 100,000). As well as stated by Mackinny [11]; where the male to female ratio was 1.5:1.0.

The incidence of BTs among both gender have specific anatomical location during 2014-2017 (Figure 3). In which the mid of the brain was the common involved anatomical segment by tumors which represents $35 \%$ followed by left brain $(30 \%)$ and the right brain as (24\%) and the least involved segment was bilateral i.e. both hemispheres of the brain. Such results are agreed with study done by Fatima, [18] in Sudan during the period 2009-2010, in which she found that: the anatomical brain tumor location in Sudanese patient were more in mid of the brain $(46.7 \%)$, left brain $(33.3 \%)$ and right $(20 \%)$. The susceptibility of mid brain to tumorigenic could be ascribed or related to physiological hormonal changes by aging, gender, occupation and habits.

While the distribution of BTs based on age groups among Sudanese population during 2014-2017, showed that: the brain tumors have been encountered even during the age groups of 1-10 years old as common brain tumors among both gender and the incidence increases following the aging which peaking at 55-65 years old then decreased rapidly after; with general similar trend among both gender and through the entire ages (Figure 4). Similar trend of BTs being distributed based on age has been highlighted by Mackinny [11]. However for 1-10 age group the common types were pediatric tumors that originated infratentorial (Medduloblastoma, astrocytoma, Glioma and Craniphyringoma) as mentioned by Wilson and Mosely, [19], while among adult; were commonly as metastasis from breast, cervix, prostate and nasopharyngeal cancers. The general picture of BTs showed that: benign tumors are more predominant among adult female; that could be ascribed to hormonal levels fluctuation and response to chemotherapy administration for several chronic inflammation, while malignant ones have been predominated among male adult.

The BTs have different types in view of histology; in Sudanese population during 2014-2017 the common pathologies of brain were atrophy and infarction that represents $13.8 \%$ and $7.2 \%$ respectively; however among these groups the impact of diabetes on brain and other vital anatomical structures as well as aging effect [20-22] increases the incidence of atrophy among adult. Then the pathologies incidence decreased for hematoma, cystic, abscess and infection while the incidence increases obviously among male and female for ischemic $(11 \%, 7.1 \%)$, inflammation $(9.1 \%$, $5.4 \%$ ), benign tumors (19.8\%) and malignant tumors (34.4\%) as in Figure (5). The possible causes for such results could be ascribed to extensive usage of underground water which is contaminated with sewage and wastewater for drinking (selfsurvey results). Also could be due to contamination by nitrate and nitrite compounds that leeching into drinking water 
supplies [23, 24] in addition to exposure to low-level radiofrequencies as accepted approved carcinogenesis [25].

The geographical distribution of BTs in different sectors of Sudan to some extend has a link with the risk factors and accordingly the the center of Sudan and North of Sudan were the most endemic sectors by BTs relative to other sectors; which represented $45 \%$ and $35 \%$ respectively, then east and south of Sudan represented $12 \%$ and $8 \%$ respectively (Figure 6). Indeed the distribution of BTs in Sudan seemed to be dependent on the population (5.2 million in 2014), aging factors: most are young adult and old ones, food and dietary factors: as has been approved that $\mathrm{N}$-nitroso compounds could induce neurosarcomas and potent neurocarcionogenic [26, 27]; in this realm Sudanese population have focused on processed meat and fast food intake abundantly, occupational and the living style: as exposed workers to electric and magnetic fields showed significant $(10 \%$ to $20 \%)$ risk increment for brain tumors [28, $29]$; in addition to the technology of communication specially wireless exemplar in cellular phones and electromagnetic field utilized in medical field (magnetic resonance imaging) as have been approved to induce certain brain vacuolation, atrophied hepatocytes, spleen and kidney rupture [30]. Relative to this facts: Sudanese population randomly living near high tension transformer/towers of electricity, wireless station and uncontrolled usage of cell phone (long duration chatting) that may contribute in increasing the risk factors and incidence of BTs.

\section{Conclusion}

BTs in Sudan have been increases annually with prominent peaking among 55-65 age groups and targeting the mid of the brain at high populated sectors in Sudan and where the risk factors of brain tumor are predominant.

\section{References}

[1] Joseph Timmons. (2012). Primary Brain Tumors - Everything a Medical Student Needs to Know. Scottish Universities Medical Journal, Dundee; 1(1): 31-37.

[2] McKinney, P A. (2004). Brain tumors: incidence, survival, and aetiology. Journal of neurology, neurosurgery and psychiatry; 75(2): 12-17. DOI: 10.1136/jnnp.2004.040741.

[3] Chandana SR, Movva S, Arora M, Singh T. (2008). Primary brain tumors in adults." American family physician. 77(10): 1423-1430. PMID: 18533376.

[4] Buckner JC1, Brown PD, O'Neill BP, Meyer FB, Wetmore CJ, Uhm JH. (2007). Central nervous system tumors. Mayo Clinic proceedings. 82(10): 1271-1286. DOI: 10.4065/82.10.1271.

[5] Stefan Lonn, Lars Klaeboe, Per Hall, Tiit Mathiesen, Anssi Auvinen, Helle C. Christensen, Christoffer Johansen, Tiina Salminen, Tore Tynes and Maria Feychting. (2004). Incidence trends of adult primary intracerebral tumors in four nordic countries. Int. J. Cancer. 108, $450-455$. DOI 10.1002/ijc. 11578 .
[6] Hussein S. Abu-Salih and Ali M. Abdul-Rahman. (1988). Tumors of the brain in the Sudan. Surgical Neurology. 3: 194196. DOI: 10.1016/0090-3019 (88) 90005-5.

[7] Intisar E Saeed, Hsin-Yi Weng, Kamal H Mohamed, and Sulma I. Mohammed. (2014). Cancer incidence in Khartoum, Sudan: first results from the Cancer Registry, 2009-2010. Cancer Med. 3(4): 1075-1084. DOI: 10.1002/cam4.254.

[8] Mohamed E. M. Saeed, Jingming Cao, Babikir Fadul, Onat Kadioglu, Hassan E. Khalid, Zahir Yassin, Siddig M. Mustafa, Elfatih Saeed and Thomas Efferth. (2016). A Five-year Survey of Cancer Prevalence in Sudan. Anticancer Research 36: 279286. PMID: 26722054.

[9] Wrensch, M., Minn, Y., Chew, T., Bondy, M., \& Berger, M. S. (2002). Epidemiology of primary brain tumors: current concepts and review of the literature. Neuro-oncology. 4(4): 278-299.

[10] Margaret Wrensch, Yuriko Minn, Terri Chew, Melissa Bondy, and Mitchel S. Berger. (2002). Epidemiology of primary brain tumors: Current concepts and review of the literature. NeuroOncology. 4: 278-299.

[11] McKinney P. A. (2004). Brain tumours: incidence, survival, and aetiology. J NeurolNeurosurg Psychiatry; 75(Suppl II):ii12-ii17. DOI: 10.1136/jnnp.2004.040741

[12] Karak, A. K., Singh, R., Tandon, P. N., and Sarkar, C. (2000). A comparative survival evaluation and assessment of interclassi. cation concordance in adult supratentorial astrocytic tumors. Pathol. Oncol. Res. 6, 46-52.

[13] Greig NH, Ries LG, Yancik R, Rapoport S. I. (1990). Increasing annual incidence of primary malignant brain tumors in the elderly. J. Natl. Cancer Inst. 82(20):1621-4. PMID: 2213902.

[14] Sun T, Warrington NM, Luo J, Brooks MD, Dahiya S, Snyder SC, Sengupta R, Rubin JB. (2014). Sexually dimorphic RB inactivation underlies mesenchymal glioblastoma prevalence in males. J. Clin. Invest. 124(9): 4123-33. DOI: 10.1172/JCI71048.

[15] Michael C. Purdy. (2014). Study reveals one reason brain tumors are more common in men. Washington University School of Medicin. https://source.wustl.edu/2014/08/studyreveals-one-reason-brain-tumors-are-more-common-in-men/ (July 13, 2017).

[16] James, C. D., Smith, J. S., and Jenkins, R. B. (2002). Genetic and molecular basis of primary central nervous system tumors. In: Levin, V. (Ed.), Cancer in the Nervous System. $2^{\text {nd }}$. Edition, New York: Oxford University Press. pp. 239-251.

[17] Central Brain Tumor Registry of the United States (CBTRUS). (2005). Statistical Report: Primary brain tumors in the United States, 1998-2002. Hinsdale, IL: Central Brain Tumor Registry of the United States.

[18] Fatima Salih, S. (2011). Brain Tumors among Sudanese Patients (A histopathological Study) (Doctoral dissertation, U of K), Sudan.

[19] Wilson J. L, I. F. Mosely. (1978). Diagnostic approach to cerebellar lesions. Neuroradiol. 4: 250-258.

[20] Hughes, T. M., C. M. Ryan, H. J. Aizenstein, et al. (2013). Frontal gray matter atrophy in middle aged adults with type 1 diabetes is independent of cardiovascular risk factors and diabetes complications. J. Diab. Compl. 27: 558-564. 
[21] Mohammed A. Ali Omer, Emad M. Mukhtar Alasar, Mohamed E. M. Gar-elnabi, Ghada A. E. Sakin, Yahia M. Bushara. (2014). Measurement of Cranial and Brain Ventricle Volumes Relative to Pathologies. International Journal of Science and Research (IJSR) 3(5): 987-991.

[22] Mohammed A. Ali Omer, Ayman H. Eljack1, Mohamed E. M. Gar-alnabi, Mustafa Z. Mahmoud, Muatasim Elseid, Ghada A. Edam. (2014-b). Ultrasonographic Characteristics of Diabetes Impacts in Kidneys' Morphology, Open Journal of Radiology. 4, 301-308. DOI:10.4236/ojrad.2014.44039.

[23] Mueller BA, Newton K, Holly EA, Preston-Martin S. (2001). Residential water source and the risk of childhood brain tumors. Environ Health Perspect. 109(6): 551-556. [PubMed: 11445506].

[24] Steindorf K, Schlehofer B, Becher H, Hornig G, Wahrendorf J. (1994). Nitrate in drinking water. A case control study on primary brain tumors with an embedded drinking water survey in Germany. Int. J. Epidemiology. 23(3):451-457. [PubMed: 7960368].

[25] Moulder JE, Foster KR, Erdreich LS, McNamee JP. (2006). Mobile phones, mobile phone base stations and cancer. Int. J. Radiation Biol. 81: 189 - 203. [PubMed: 16019928].

[26] Dietrich, M., Block, G., Pogoda, J. M., Buffler, P., Hecht, S.,
\& Preston-Martin, S. (2005). A review: Dietary and endogenously formed $\mathrm{N}$-nitroso compounds and risk of childhood brain tumors. Cancer Causes and Control. 16(6), 619-635. DOI: 10.1007/s10552-005-0168-y.

[27] Yun Zhu, Peizhong Peter Wang, Jing Zhao, Roger Green, Zhuoyu Sun, Barbara Roebothan, Josh Squires, Sharon Buehler, Elizabeth Dicks, Jinhui Zhao, Michelle Cotterchio, Peter T. Campbell, Meera Jain, Patrick S. Parfrey, and John R. Mclaughlin. (2014). Dietary N-nitroso compounds and risk of colorectal cancer: a case-control study in Newfoundland and Labrador and Ontario, Canada. Br. J. Nutr. 28; 111(6): 1109 1117. DOI: $10.1017 / \mathrm{S} 0007114513003462$.

[28] Zhuoyu Sun, Barbara Roebothan, Josh Squires, Sharon Buehler, Elizabeth Dicks, Jinhui Zhao, Michelle Cotterchio, Peter T. Campbell, Meera Jain, Patrick S. Parfrey, and John R. Mclaughlin. (2014). Dietary N-nitroso compounds and risk of colorectal cancer: a case-control study in Newfoundland and Labrador and Ontario, Canada. Br. J. Nutr. 28; 111(6): 1109 1117. DOI: $10.1017 / \mathrm{S} 0007114513003462$.

[29] Kheifets L I, Afifi A A, Buffler P A, Zhang Z W, (1995). Occupational electric and magnetic field exposure and brain cancer: a meta-analysis. J. Occup. Environ Med. 35:1327-41.

[30] Hans-Olov Adami, David Hunter, Dimitrios Trichopoulos. (2008). Textbook of Cancer Epidemiology, $2^{\text {nd }}$. edition, Oxford University Press, New York - USA. 